PROCEEDINGS OF THE

AMERICAN MATHEMATICAL SOCIETY

Volume 132, Number 11, Pages 3345-3347

S 0002-9939(04)07445-3

Article electronically published on April 21, 2004

\title{
DESCRIPTIVE PROPERTIES OF THE SET OF EXPOSED POINTS OF COMPACT CONVEX SETS IN $\mathbb{R}^{3}$
}

\author{
PETR HOLICKÝ AND MIKLÓS LACZKOVICH \\ (Communicated by Carl G. Jockusch, Jr.)
}

\begin{abstract}
We construct a compact convex subset of $\mathbb{R}^{3}$ such that the set of its exposed points is not the intersection of an $F_{\sigma}$ set and a $G_{\delta}$ set. The existence of such a set answers a question posed by G. Choquet, H.H. Corson and V.L. Klee.
\end{abstract}

Let us recall that $x \in C$ is called an exposed point of the convex set $C \subset \mathbb{R}^{n}$ if there is a real linear functional $f$ on $\mathbb{R}^{n}$ such that $f(y)<f(x)$ for all $y \in C, y \neq x$. Clearly, $x$ is an exposed point of $C$ if and only if $\{x\}=H \cap C$ for some supporting hyperplane $H$ of $C$. The set of all exposed points of $C$ is denoted by $\exp C$. We use conv $A$ to denote the convex hull of $A$, and $\overline{\operatorname{conv}} A$ to denote its closure.

It was shown in [1 Theorem 1.1] that if $K$ is a compact convex set in $\mathbb{R}^{3}$, then $\exp K$ is the union of a $G_{\delta}$ set and a set which is the intersection of a $G_{\delta}$ set and an $F_{\sigma}$ set.

V.L. Klee [3] constructed a compact convex set $K$ in $\mathbb{R}^{3}$ such that $\exp K$ is not a $G_{\delta}$ set. H.H. Corson [2] gave an example of a compact convex set $K$ in $\mathbb{R}^{3}$ such that $\exp K$ is not even the union of a $G_{\delta}$ set and an $F_{\sigma}$ set.

The aim of our remark is to answer in the negative the following question posed by G. Choquet, H. Corson and V. Klee [1, Problem 1.2]: if $K$ is a three-dimensional compact convex set, must $\exp K$ be the intersection of a $G_{\delta}$ set and an $F_{\sigma}$ set?

Theorem 1. Given a set $E=F \cup G \subset S=\left\{x \in \mathbb{R}^{2} ;\|x\|=1\right\}$ such that $F$ is an $F_{\sigma}$ subset of $S$ and $G$ is a $G_{\delta}$ subset of $S$, then there is a compact convex set $C \subset \mathbb{R}^{3}$ with $\left\{x \in \mathbb{R}^{2} ;(x, 0) \in \exp C\right\}=F \cup G$.

The following lemma is probably known; however, we were not able to find a reference. We shall provide the simple proof.

Lemma 2. There are an $F_{\sigma}$ subset $F$ of $S$ and a $G_{\delta}$ subset $G$ of $S$ such that their union $F \cup G$ is not the intersection of an $F_{\sigma}$ set and a $G_{\delta}$ set.

Received by the editors February 5, 2003 and, in revised form, July 21, 2003.

2000 Mathematics Subject Classification. Primary 52A15, 28 A05.

Key words and phrases. Exposed points, convex compact sets.

The first author was supported by the "Mathematics in Information Society" project carried out by Alfréd Rényi Institute of Mathematics, Hungarian Academy of Sciences, in the framework of the European Community's "Confirming the International Role of Community Research" program. The research was partly supported also by grants GAČR 201/03/0931 and MSM 113200007.

The second author was partially supported by the Hungarian National Foundation for Scientific Research Grant No. T032042. 
If we apply Theorem 1 with the set constructed in Lemma 2 we obtain a negative answer to the problem of G. Choquet, H. Corson and V. Klee.

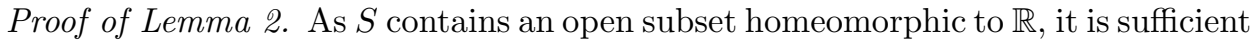
to look for the required sets in $\mathbb{R}$. Also, as $\mathbb{R}$ contains a homeomorphic copy of the Cantor set $C=\{0,1\}^{\mathbb{N}}$, we may work in $C$. Finally, as the product $C^{2}=C \times C$ is homeomorphic to $C$, it is enough to find the required sets in $C^{2}$.

Let $Q$ be a countable dense subset of $C$. Then $Q^{2}$ is an $F_{\sigma}$ set and $(C \backslash Q)^{2}$ is a $G_{\delta}$ set. We claim that $Q^{2} \cup(C \backslash Q)^{2}$ is not the intersection of an $F_{\sigma}$ set and a $G_{\delta}$ set.

Suppose that $Q^{2} \cup(C \backslash Q)^{2}=E \cap H$, where $E$ is $F_{\sigma}$ and $H$ is $G_{\delta}$ in $C^{2}$. As $E$ contains the second category set $(C \backslash Q)^{2}$, it follows that $E$ itself is of second category in $C^{2}$ and, being an $F_{\sigma}$ set, its interior in not empty. Suppose $U \times V \subset E$, where $U$ and $V$ are nonempty open subsets of $C$. Let $x \in U \cap Q$ be fixed. Then we have $(\{x\} \times Q) \cap(\{x\} \times V)=H \cap(\{x\} \times V)$, and thus $Q \cap V$ is a $G_{\delta}$ set, which is not the case.

Proof of Theorem 1. Let $F=\bigcup_{n \in \mathbb{N}} F_{n}$ and $S \backslash G=\bigcup_{n \in \mathbb{N}} E_{n}$, where the sets $F_{n}$, $E_{n}(n \in \mathbb{N})$ are closed.

For $x \in S$ and $n \in \mathbb{N}$, let $f_{x, n}$ be the real linear functional on $\mathbb{R}^{3}$ such that $f_{x, n}(0,0, n)=1, f_{x, n}(x, 0)=1$ and $f_{x, n} \leq 1$ on $S$. Put $H_{x, n}=f_{x, n}^{-1}((-\infty, 1])$. Then $H_{x, n}$ is a half-space such that its boundary $\partial H_{x, n}$ touches the circle $S$.

Using the notation $B_{+}=\left\{(x, y) \in \mathbb{R}^{2} \times \mathbb{R}=\mathbb{R}^{3} ;\|(x, y)\| \leq 1, y \geq 0\right\}$ and $B_{-}=\left\{(x, y) \in \mathbb{R}^{2} \times \mathbb{R} ;(x,-y) \in B_{+}\right\}$, we define $C=C_{+} \cup C_{-}$, where

$$
C_{+}=B_{+} \cap \bigcap_{n \in \mathbb{N}} \bigcap_{x \in F_{n}} H_{x, n}
$$

and

$$
C_{-}=\overline{\operatorname{conv}}\left(B_{-} \cup\left(\bigcup_{n=1}^{\infty} E_{n} \times\left[-\frac{1}{n}, 0\right]\right)\right) .
$$

Clearly, $C$ is compact, and it is convex since the convex sets $C_{+}$and $C_{-}$satisfy

$$
\begin{aligned}
& C_{+} \subset\left\{(x, y) \in \mathbb{R}^{2} \times \mathbb{R} ;\|x\| \leq 1, y \geq 0\right\}, \\
& C_{-} \subset\left\{(x, y) \in \mathbb{R}^{2} \times \mathbb{R} ;\|x\| \leq 1, y \leq 0\right\},
\end{aligned}
$$

and

$$
C_{+} \cap C_{-} \supset\left\{(x, 0) \in \mathbb{R}^{2} \times \mathbb{R} ;\|x\| \leq 1\right\} .
$$

It remains to show that $\exp C \cap(S \times\{0\})=(F \cup G) \times\{0\}$.

To show that $F \times\{0\} \subset \exp C$, suppose that $z_{0}=\left(x_{0}, 0\right) \in F_{n} \times\{0\}$ for some $n \in \mathbb{N}$. Then the supporting plane $\left\{(x, 2 y) \in \mathbb{R}^{2} \times \mathbb{R} ; f_{x_{0}, n}(x, y)=1\right\}$ meets $C$ in the single point $z_{0}$, thus $z_{0} \in \exp C$.

Let $x_{0} \in G$ and $z=\left(x_{0}, y\right) \in C$. We shall show that necessarily $y=0$, and so the point $z_{0}=\left(x_{0}, 0\right)$ is an exposed point of $C$ (it is even an exposed point of the set $\left\{(x, y) \in \mathbb{R}^{2} \times \mathbb{R} ;\|x\| \leq 1, x \neq x_{0}\right\} \cup\left\{\left(x_{0}, 0\right)\right\}$ which contains $\left.C\right)$. Clearly $y \leq 0$, as $C_{+} \subset B_{+}$. In what follows we are using the fact that the convex hull of a compact subset of $\mathbb{R}^{3}$ is compact (and thus closed) [4, Theorem 17.2]. For an arbitrary $n \in \mathbb{N}$ we have

$$
C_{-}=\operatorname{conv}\left(\operatorname{conv}\left(B_{-} \cup \bigcup_{k=1}^{n}\left(E_{k} \times\left[-\frac{1}{k}, 0\right]\right)\right) \cup \overline{\operatorname{conv}}\left(\bigcup_{k=n}^{\infty}\left(E_{k} \times\left[-\frac{1}{k}, 0\right]\right)\right)\right) .
$$


Thus $z=\left(x_{0}, y\right)=\alpha_{1} \cdot\left(x_{1}, y_{1}\right)+\alpha_{2} \cdot\left(x_{2}, y_{2}\right)$, where $\alpha_{1}, \alpha_{2}$ are nonnegative reals with $\alpha_{1}+\alpha_{2}=1,\left(x_{1}, y_{1}\right) \in \operatorname{conv}\left(B_{-} \cup \bigcup_{k=1}^{n}\left(E_{k} \times\left[-\frac{1}{k}, 0\right]\right)\right)$ and $\left(x_{2}, y_{2}\right) \in$ $\overline{\mathrm{conv}} \bigcup_{k=n}^{\infty}\left(E_{k} \times\left[-\frac{1}{k}, 0\right]\right)$. Since $\left\|x_{0}\right\|=1$ and $\left\|x_{i}\right\| \leq 1(i=1,2)$, it follows that $x_{0}=x_{1}=x_{2}$. Thus $y_{1}=0$, as $B_{-} \cap(S \times(-\infty, 0))=\emptyset$, and $x_{1}=x_{0} \in G \subset$ $S \backslash \bigcup_{k=1}^{n} E_{k}$. Therefore $y=\alpha_{2} y_{2} \geq \alpha_{2} \cdot\left(-\frac{1}{n}\right) \geq-1 / n$, and, as $n \in \mathbb{N}$ was arbitrary, we have $y=0$.

To prove the other inclusion, we suppose that $x \in S \backslash(F \cup G)$ is such that $(x, 0) \in \exp C$, and we proceed to a contradiction. As $x \notin G$, it belongs to $E_{n_{0}}$ for some $n_{0} \in \mathbb{N}$. Thus $\{x\} \times\left[-\frac{1}{n_{0}}, 0\right] \subset C$, and necessarily $\partial H_{x, n_{1}} \cap C=f_{x, n_{1}}^{-1}(1) \cap C=$ $\{(x, 0)\}$ for some sufficiently large $n_{1} \in \mathbb{N}$.

On the other hand, we shall prove that for any $n \in \mathbb{N}$ (we shall use it for $n=n_{1}$ ) there is an $\varepsilon>0$ such that

$$
B_{+} \cap \operatorname{conv}((S \times\{0\}) \cup\{(0,0, n)\}) \cap \operatorname{conv}\{(x, 0),(x, \varepsilon),(0,0,1)\} \subset C .
$$

Suppose for a while that (11) is proved. Then we make the observation that there is an element distinct from $(x, 0)$ in the set

$$
\partial H_{x, n_{1}} \cap B_{+} \cap \operatorname{conv}\left((S \times\{0\}) \cup\left\{\left(0,0, n_{1}\right)\right\}\right) \cap \operatorname{conv}\{(x, 0),(x, \varepsilon),(0,0,1)\} \cap C,
$$

which contradicts the fact that $f_{x, n_{1}}^{-1}(1) \cap C=\{(x, 0)\}$.

It remains to prove (1). As $\operatorname{dist}\left(x, \bigcup_{k=1}^{n} F_{k}\right)>0$, there is an $\varepsilon>0$ such that

$$
\bigcap_{k=1}^{n} \bigcap_{x^{\prime} \in F_{k}} H_{x^{\prime}, k} \supset \bigcap_{k=1}^{n} \bigcap_{x^{\prime} \in F_{k}} H_{x^{\prime}, 1} \supset \operatorname{conv}\{(x, 0),(x, \varepsilon),(0,0,1)\} \text {. }
$$

Also

$$
\bigcap_{k=n}^{\infty} \bigcap_{x^{\prime} \in F_{k}} H_{x^{\prime}, k} \supset \bigcap_{k=n}^{\infty} \bigcap_{x^{\prime} \in F_{k}} H_{x^{\prime}, n} \supset \operatorname{conv}((S \times\{0\}) \cup\{(0,0, n)\}) .
$$

Now the inclusion (1) follows from the definition of $C_{+}$.

\section{REFERENCES}

[1] G. Choquet, H. Corson and V. Klee, Exposed points of convex sets, Pacific J. Math. 17 (1966), 33-43. MR 33:6335

[2] H.H. Corson, A compact convex set in $E^{3}$ whose exposed points are of the first category, Proc. Amer. Math. Soc. 16 (1965), 1015-1021. MR 31:5147

[3] V.L. Klee, Extremal structure of convex sets, II, Pacific J. Math. 69 (1958), 90-104. MR 19:1065b

[4] R.T. Rockafellar, Convex Analysis, Princeton, New Jersey, Princeton University Press 1970. MR 43:445

Department of Mathematical Analysis, Charles University, Sokolovská 83, 18695 Prague 8, Czech Republic

E-mail address: holicky@karlin.mff.cuni.cz

Department of Analysis, Eötvös Loránd University, Budapest, Pázmány Péter sétány 1/C, Hungary - and - Department of Mathematics, University College London, Gower Street, London WC1E 6BT, England

E-mail address: laczk@cs.elte.hu 